\title{
Exploring Motivations of Taekwondo Athletes/Students in New York City
}

\author{
Howard Z. Zeng ${ }^{1, *}$, Wojciech. J. Cynarski ${ }^{2}$, Shannon Baatz ${ }^{1} \&$ Shawn J. Park $^{1}$ \\ ${ }^{1}$ Department of Kinesiology, Brooklyn College of the City University of New York, New York, USA \\ ${ }^{2}$ Department of Sport Study, University of Rzeszów, Rzeszów, Poland \\ *Correspondence: 2900 Bedford Ave. Brooklyn, NY, 11210, USA. Tel: 1-718-318-7477. E-mail: \\ hzeng@brooklyn.cuny.edu
}

Received: August 8, 2015

Accepted: September 24, $2015 \quad$ Online Published: October 7, 2015

doi:10.5430/wje.v5n5p51

URL: http://dx.doi.org/10.5430/wje.v5n5p51

\begin{abstract}
This study explored what reasons/factors can truly motivate athletes/students to continually participate in taekwondo practices and competitions in New York City (NYC). Participants were 85 taekwondo athletes/students (51 Boys, 34 Girls; age range 10-22 years) from seven taekwondo schools/clubs in NYC. Data collection was done by the Adapted Questionnaire of Taekwondo Student's Motivation (AQTKDSM). This questionnaire asked, "What reasons/factors motivate you to participate in taekwondo practices and competitions continually?" with 18 motivation factors (MF) provided; each MF allows the participant responding in a 5-points Likert type scale (5 represents "Strongly-fit" and 1 represents "Not-it"). Data analyses included descriptive statistics and a 2 x 4 (Gender [males, females] x Belt-Colors [yellow, blue, red, black]) multivariate analysis of variance (MANOVA). Findings included: The top three reasons/factors were 'Technical content and unique value', 'For fun' and 'Shape body' $(M=$ 4.79 to $M=4.24$ ); these three MF possessed the highest impact power on their motivation. The results of 2 × 4 MANOVA revealed: significant differences were found in both 'Gender' and 'Belt-colors'; e.g., males scored over females in 'Shape body' and 'Become a professional'; females scored over males in 'Establish prestige' and 'Self-defense'. As to Belt-Colors comparisons, Black scored over Red in 'technical content and unique value'; Blue over Yellow in 'For fun' and Black scored over Red in 'Make new friends'. In conclusion, the most important motivation reasons/factors are: 'Value', 'Enjoyment', 'Self-esteem', and 'Physical needs' four intrinsic motivations; and 'Develop skills', 'Establish prestige', 'Build-up friendship', and 'Contest winners' four extrinsic motivations.
\end{abstract}

Keywords: motivation; taekwondo; athletes/students; physical health; self-esteem

\section{Introduction}

Taekwondo scientific literatures mainly focus on the sports dimension and all that affects the outcome of this competitive sport (e.g., Pieter \& Heijmans 2000; Capranica et al. 2010). According to Stefanek (2004) and Wasik (2014), research studies covered and reflected motivations of taking part in taekwondo are still quite limited; generally speaking, however, the purposes or reasons of taking part in taekwondo practices and competitions are: 'self-defense', 'education', 'enjoyment' and 'physical health'. The last two reasons are similar to the dominant Western society's recreational activity (e.g., Cox, 2011; Clough, Shepherd \& Maughan, 1989; Devine \& Lepisto, 2005; Duda, 1989; Gill, Gross \& Huddleston, 1983).

Studies on taekwondo practices and competitions found that only $20 \%$ of adults were interested in attending taekwondo competition whereas up to $60 \%$ of youth enjoy taking part in both the practices and competitions (Hartl, Faber, Bögle, 1989). Sports competition is something attractive to young people and to various sports organizers as well. Cynarski (2014a) explained that the reasons why people take part in martial arts practices and competitions are related to: Kara means void, to empty, and to purify of selfishness and anger; and signifies clear mind, heart and hands as well. According to General Choi - the founder of International Taekwondo Federation (ITF) this explanation suitable for both karate and Taekwondo (Cynarski, 2014a).

Moreover, according to the creator of taekwondo - Choi, Hong Hi, taekwondo is just a Korean variation of the karate (Bujak 2001c), which is confirmed by martial arts historians (Rzany, 2002-2003; Madis, 2003). Therefore, the 
motivations to participate in the activities of taekwondo are very similar to those found in the studies of motivation among the karate athletes. In terms of its specificities, there are some differences between the International Taekwon-do Federation (ITF) and the World Taekwondo Federation (WTF); the ITF style is closer to karate-do, as a martial art (Choi, 1983, 1995; Bujak, 2001a, b, 2005; Wasik, 2014), and accents relevance of the route of self-improvement; this style applies to the process of physical education (Walczak, 2007); in order to represent ITF, and mark its distinctiveness from the karate, the name of 'taekwon-do' was used. On the other hand, the WTF is more like a combat sport, and is an Olympic discipline. The leaders of WTF were trying to demonstrate the originality of the sport (e.g., Lee, Nowicki, 1988; Tedeschi, 2004); so they named this sport as taekwondo. The taekwondo variety is the most widely studied as a competitive sport (e.g., Capranica et al., 2010; Pieter \& Bercades, 2010). Because none of the participants in the currents study were professional taekwondo athlete/students, and the words of taekwondo appears in the research articles more frequently, we would just simply adopt the name of taekwondo instead of the taewon-do.

According to researchers and scholars of martial arts, there was a set of ethical and utilitarian values that formed the specificity of martial arts: As ethical systems martial arts develop 'good man' (Choi 1983; Kim D. \& Back 2000; Kim S., 2010; Cynarski, 2012; Cynarski, Obodynski \& Zeng, 2012). This idea is also suitable in 'personal models' (Bujak, 2001c; Kim, 2010). These unique values affected the motivation of practicing different varieties of martial arts and combat sports; so is in the sport of taekwondo - a branch of martial arts (e.g., Litwiniuk \& Cynarski, 2001; Stefanek, 2004; Jones, Mackay, Peters, 2006; Vertonghen, Theeboom, 2010; Kusnierz, 2011; Vit, Reguli, 2011; Rosa, 2012; Witkowski, Cynarski, \& Blazejewski, 2013). Specifically, Cynarski (2012) and Litwiniuk and Cynarski (2001) indicated that the factors that motivate young people to take part in martial arts practices are both conscious and unconscious. The conscious ones have the desire to improve physical fitness, acquire fighting skills, gain a sense of security, meet interesting people, and sports competition. While the unconscious include: the influence of a hero-figure, the needs of personal security, acceptance by the martial arts group or community, and to prove a self-concept or self-encouragement that 'I can'.

An expressive example on martial arts students' motivation study in the USA should be the study of "An analysis of student's reasons for studying martial arts" by Twemlow, Lerma and Twemlow (1996). Their study determined the initial reasons why students took part in martial arts practices and competitions. Findings included: Over $90 \%$ of the participants reported 'self-defense' was their main reason for engaging in martial arts practices and competition. Other than that the researchers fund that there are more essential reasons for those participants who participate in practices and competitions continually, and the researchers named those essential reasons as the 'subtle psychological motivations' which are an individual's wish for power and to achieve almost magical abilities (Twemlow, Lerma, \& Twemlow, 1996). These researchers summarized that: self discipline, self-defense, outlet for aggression, physical exercise, social support, development of self-esteem, self-confidence, power, and mastery of skills are the reasons or factors for martial arts students participate in, and continually engage in martial arts. Hence, they suggested that martial clubs / schools should cater those unstated needs that may cause many students to drop out when their needs are not achieved. In order to achieve these needs, instructors / coaches must develop abilities to transform their athlete's or student's needs to the power and magical abilities, broadening their mental skills as well as self-development.

Because most of the martial arts and combat sports benefit the physiological elements of the participant, motivations studies in martial arts are frequently treated as health and fitness activities (e.g., Kilpatrick, Hebert \& Bartholomew 2005; Zeng, Cynarski \& Xie, 2013). Kusnierz (2011) in his martial arts motivation study summarized: Facts that the essential motivate for engaging in training was to master the hand-to-hand fighting and self-defense rules and skills. The martial arts training increased participants' discipline and the level of fitness and health. On the other hand, for many people, the social aspect of the martial arts is also an important reason for their participation. From this aspect, Reis (2005) even declared that to take part in martial arts practices can also improve the well being of a society.

\subsection{Theoretical Framework}

The theoretical frameworks that guide the current study are the theory of intrinsic and extrinsic motivation. With regard to motivation theoretical framework Ryan and Deci (2000) pointed out, there is a comprehensive theoretical framework called the 'self-determination theory' (SDT); Ryan and Deci clearly stressed that humans are motivated by three basic psychological needs: competence, relatedness, and autonomy. Ryan and Deci further explained, the competence needs in SDT model is called effectiveness motivation; the relatedness needs refers to people's need to belong and to feel accepted by others; and the autonomy need, however, refers to people's need to feel self-determined, it is the source of their own action. 
Similarly, Harter (1981), and Pintrich and Schunk (2002) described that organismic needs energize intrinsic and extrinsic motivations, but believe this concept is too general to explain engagement in specific behaviors (e.g., sport behaviors) and too vague to be a guide for empirical research. Researchers, therefore, developed a few models that described how motivation triggered by needs manifests in intrinsic and extrinsic motivation in specific domains and activities. These models also explained how factors in a specific environment might shape and affect the types of motivation that people manifest in different activities (Harter, 1981; Pintrich \& Schunk, 2002).

Specifically, Breese (1998) pointed out, athletics initial motivation should be defined as intrinsic motivation (participating in sport for enjoyment) or extrinsic motivation (participating in sport to gain rewards). Breese continued, athletics initial motivation usually predicts athletes' attendance and adherence to a particular sport. Such as in the present study, a taekwondo athlete/student who is intrinsically motivated would be those who go to practice his or her skills or forms every other day for fun; whereas a taekwondo athlete/student who is extrinsically motivated would be those who goes to practice his or her taekwondo skills to become a better performer at the competition so that he/she could win a medal at a competition. It is interesting to know that intrinsic and extrinsic motivation have different effects on an athlete, including whether or not he/she continue on the sport he/she had choose.

Additionally, Ryan, Frederick, Lepes, Rubio and Sheldon (1997) explained that individuals who were mainly motivated by competence (engaging in exercise to expand skills) and enjoyment (the desire to have fun) could be primarily defined as being motivated intrinsically. In contrast, extrinsically motivated individuals are those behaviors performed in intrinsic motivation aim at to obtain rewards or consequences that are separate from the behavior itself. Breese (1998) also illustrated that when athletes begin participation in a particular sport, they are motivated not only by intrinsic factors but also extrinsic factors. Some particular sports, however, may be more relying on intrinsic motivation than extrinsic motivation (e.g., in the report of Ryan, Frederick, Lepes, Rubio and Sheldon, 1997). The reasons are: different types of sports need different types of motivation (evidences can be found in their report, Breese, 1998). In the present study, we were trying to find out those evidences or the factors that truly motivate our participants to take part in and keep engaging in the sport of taekwondo.

\subsection{Purpose and Hypotheses}

According to the introduction above, although some of the reasons (e.g., self-defense, education, and sport and health) have known in general, little is known about what kinds of factors or reasons that really motivate various types of students take part in and involve competitions in taekwondo continually in New York City (NYC). The purpose of this study, therefore, was to explore what factors or reasons that truly motivate athletes/students in difference education and skill levels participating in taekwondo practices and competitions in NYC. The following specific hypotheses guided the current study: (a) No significant differences would be found on the motivation factors or elements between the gender of the athletes/students; (b) no significant differences would be found on the motivation factors or elements between education levels of the athletes/students; (c) no significant differences would be found on the motivation factors or elements among the Belt Colors of the athletes/students who participate in taekwondo practices and competitions. The findings from this research would reveal and add first-hand information and new set of data into the field of martial arts study in regard to athletes/students' motivations.

\section{Methods}

\subsection{Sampling Procedures}

According to United States Census Bureau (2014), the population of NYC was 8,491,079 (Wikipedia, 2015). The present study was conducted during the academic year of 2013 to 2014 using the NYC taekwondo population. Approximately, there are 275 to 300 taekwondo schools/clubs in NYC. When, divided into five boroughs geographically the boroughs of Manhattan and Brooklyn were selected because their taekwondo populations combined are nearly $45 \%$ of the overall taekwondo population in the whole of NYC $\left(2013^{*}-*^{*}\right.$ based on an unofficial statistics data on numbers of taekwondo schools / clubs in NYC). Additionally, low sampling costs and access to participants were other notable reasons for selecting participants; athlete/student who under 10 years old were also excluded from the sampling due to possible problems on understanding and completing the questionnaire. As a result, 85 out of 150 taekwondo athletes/students (51 males, 34 female; age range: 10-22 years) from seven taekwondo schools / clubs completed and returned the survey voluntarily.

\subsection{Procedure}

The questionnaires were distributed and collected at schools / clubs training days, where the participants gather at the same place at the same time. The questionnaire was completed at the discretion of the respondent and collected 
within two weeks. Respondents were given instructions on how to complete and hand back the questionnaire; and were also informed of their rights of non-participation, and confidentiality. The participants were given envelopes to prevent instructors or coaches from viewing returned questionnaires and to maintain anonymity. In total, 150 questionnaires were distributed during training days. Instructors or coaches were told that they would be provided with the overall results for their schools / clubs after the research have been concluded.

\subsection{Instrumentation}

The instrumentation employed for the present study was the Adopted Questionnaire of Taekwondo Student's Motivation (AQT KDSM, Zeng, Cynarski \& Xie, 2013). The reasons for using this questionnaire for to collect data were: a) There is an existing questionnaire with similar purposes; b) funding available for this study was very limited, to develop a new questionnaire more funds and times would have been needed; c) there were specialists are available for revising key words from the exist questionnaire to specify uses for taekwondo; d) research assistants (they were also taekwondo instructors/coaches) were available for questionnaire distribution and collection.

\subsubsection{Reliability and Validity of the Instrument}

Child (1990) indicated that in order to explore the possible underlying factor of the structure of a set of measured variables without imposing any preconceived structure on the outcome, the exploratory factor analysis (EFA) is the best solution. Therefore, the EFA was executed. The results showed: the analysis extracted 6 factors with perfect correspondence to the 18 reasons / items with eigenvalues for the reasons or factors ranging from 2.45 to 8.23 and structure coefficients from .71 to .92 and the majority of the fitted residuals reached the pre set-up significant difference $(\mathrm{P}<.05)$ level. Additionally, the validation process was through a pilot study, reviewing to the content or items. These processes confirmed the following concerns: a) the readability and writing skills of the youth participants $(10-12$ years old); b) whether or not those young participants can truly read and understand and complete the questionnaire accurately; c) it may result in re-wording on some questions or statements to improve the understanding for those younger respondents; d) it may result in cutting or adding numbers of the questions or statements in the questionnaire, and e) whether or not the questions or statements have asked all the possible motivation reasons or factors for the athletes/students participation in taekwondo practices and competition.

As a result, the AQTKDSM (Zeng, Cynarski \& Xie, 2013) contained two parts: Part I asked 'General Information', containing 10 questions that cover participant's general information. Part II asked, "What reasons/factors motivate you to take part in practices and competitions continually" with 18 motivation factors (MF) provided. In each MF the participant allowed to respond in 5-points Likert type scale (number 5 represents "Strongly fit", number 4 represents "It fits", number 3 represents "Somewhat-fit", number 2 represents "Little-fit", and number 1 represents "Not-fit"). To summarize, there are nine intrinsic factors (i.e., items $1,2,6,7,9,13,14,15, \& 18$ ); nine extrinsic factors (i.e., items $3,4,5,8,10,11,12,16, \& 17$ ) in the AQTKDSM; it also included the three basic psychological needs (i.e., competence, relatedness, and autonomy) described by Ryan and Deci (2000). The entire AQTKDSM (Zeng, Cynarski \& Xie, 2013) can be found in Appendix A.

\subsection{Research Design and Data Analyze Techniques}

The goal for the data analyze was to look for effects of three independent variables on 18 dependent variables. That is: Gender (male and female) x Education Level (Elementary, Middle-School, High-School and College) x Belt Colors (Green; Purple, Red, and Black) at the same time. Therefore, the data analyses included a $2 \times 4 \times 4$ (Gender [male, female] x Education levels [Elementary, Middle-School, High-School, College] x Belt-Colors [Green; Purple, Red, Black] factorial multivariate analysis of variance (MANOVA)] and after significant differences findings there was a follow up MANOVA test. Descriptive statistics were utilized to reflect the general status of how the participants were motivated participating in taekwondo practices and competition. The $2 \times 4 \times 4$ MANOVA was utilized to examine whether or not there are significant differences exist among the three independent variables and the 18 dependent variables. The follow up MANOVA test were used for determining what differences exactly exist among the dependent variables. The statistical program used for the data analyses was IBM Statistical Package for the Social Sciences (SPSS) Version 22.

\section{Results}

\subsection{Results of General Information of the Participants}

The following section presents the findings of the current study. It is structured to address the reasons / factors the participants engage in the sport of taekwondo. In total of the 150 questionnaires distributed, 85 were returned and completed correctly; this represents a response rate of $56.7 \%$. Data in Table 1 reflected "General Information of the 
participants". For example, the participants self-reported that they officially take part in taewondo study were "One year $=11.8 \%$, Two years $=25 \%$, Three years $=33 \%$, Four year and more $=42 \%$ " separately; their belt Colors* were: Yellow - Orange $=15 \%$, Green - Purple $=39 \%$, Red - Brown $=29 \%$, Black 1, 2, \& 3 $=16 \%$ separately". (Note that. In order to simplify the writing we used the first Color of the above dual belts to represent the skill levels; that is: Yellow, Green, Red, and Black).

Table 1. General Information of the Taekwondo Athletes / Students ( $N=85,51$ Males, 34 Females)

\begin{tabular}{lll}
\hline Number / Questions & Answers / Frequency / Percentage & \\
\hline $\begin{array}{l}\text { 1. What school level are } \\
\text { you currently going to? }\end{array}$ & Elementary school $=21$ or $24.7 \%$ & Middle school $=15$ or $17 \%$ \\
2. How long have you officially & College $\&$ up $=34$ or $40.0 \%$ \\
Learnt/practiced taekwondo? $=15$ or $17.7 \%$ & Two years $=21$ or $25 \%$ \\
$\begin{array}{c}\text { 3. Is your parents support you } \\
\text { learn/practice taekwondo? }\end{array}$ & Three years $=28$ or $33 \%$ & Four year and $+=36$ or $42 \%$ \\
4. What is your belt color? & Strongly support $=35$ or $41.2 \%$ & Support $=33$ or $39 \%$ \\
& Yellow - Orange $=13$ or $15.3 \%$ & Blue - Purple $=33$ or $39 \%$ \\
5. How many times do you & Red - Brown $=25$ or $29.4 \%$ & Black $1,2, \& 314$ or $=16 \%$ \\
practice in a week? & One time $=0$ & Two times $=39$ or $46 \%$ \\
6. Where do you live during your & Three times $=34$ or $40.0 \%$ & Four to five times $=12$ or $14 \%$ \\
Taekwondo training/practising? & Dormitory $=0$ & Boarding-school $=15$ or $18 \%$ \\
\end{tabular}

*Note. According to "Taekwondo Belt System" (http://www.taekwondo-network.com/tae-kwon-do-belt-system.html), "Taekwondo color belts are a system to determine where a student resides in their studies." There are 10 Colors in the system: White, Yellow, Orange, Green, Blue/Purple, Brown, Red/Bodan, and black. "The Colors themselves are progressive and associated with philosophical meaning." For the purpose of the present study and the reality of our participants' belt color distribution we have re-divided by their "Belt-colors) into the following four categories: Yellow (cover White), Blue (cover Orange, Green, and Purple), Red (cover Brown, and Bodan), and Black (cover all levels in Black). See Table 3 also.

\subsection{Means and Standard Deviations on Factors/Reasons that Motivated the Participants Took-part in Taekwondo}

Table 2. Means and Standard Deviations for All Factors / Reasons that Motivated the Participants Initiate $(N=85$, 51 Males, 34 Females)

\begin{tabular}{|c|c|c|}
\hline Factors of Motivation & $M \pm S D$ & Sum \\
\hline F1. Because taekwondo with high technical content and unique value. & $4.788 \pm .439$ & 407.00 \\
\hline For the fun and get rid of boredom. & $4.258 \pm .833$ & 362.00 \\
\hline In order to meet friends. & $3.717 \pm 1.332$ & 316.00 \\
\hline In order to make new friends. & $3.752 \pm .843$ & 319.00 \\
\hline In order to contest winners. & $3.752 \pm 1.016$ & 319.00 \\
\hline In order to shape the body. & $4.235 \pm .781$ & 60.00 \\
\hline In order to improve physical health. & $4.211 \pm 1.167$ & 358.00 \\
\hline For the near future may become a taekwondo professional. & $3.223 \pm 1.374$ & 274.00 \\
\hline In order to foster self-esteem. & $4.270 \pm .822$ & 363.00 \\
\hline F10. In order to improve my own literacy. & $3.447 \pm 1.149$ & 293.00 \\
\hline F11. In order to establish prestige among my classmates / friends. & $3.917 \pm 1.060$ & 333.00 \\
\hline F12. In order to get the recognition from my teacher / coach. & $3.658 \pm .982$ & 311.00 \\
\hline F13. In order to reduce the learning / working pressure. & $2.964 \pm 1.417$ & 252.00 \\
\hline F14. In order to reduce the troubles from learning / work. & $3.411 \pm 1.311$ & 290.00 \\
\hline F15. In order to develop the skills. & $4.129 \pm 1.261$ & 351.00 \\
\hline F16. Hope to become taekwondo coach in near future. & $3.658 \pm 1.291$ & 264.00 \\
\hline F17. In order to satisfy the will of family. & $2.400 \pm 1.155$ & 204.00 \\
\hline F18. For self-defence & $3.458 \pm .945$ & 294.00 \\
\hline
\end{tabular}

Note. 1) Factor (F) 1, 2, 6, 7, 9, 13, 14, 15, and 18 are 'Intrinsic motivation factors'; and F 3, 4, 5, 8, 10, 11, 12, 16, and 17 are 'Extrinsic motivation factors'. 2) F1, F9, F2, F7, \& F15 scored on the top; F10, 14, F8, F13, \& F17 scored on the bottom. 
Data in Table 2 reflected the "Factors that motivated the participants to initially take part in, keep practices and continuing engage in taekwondocompetition". As can be seen, the top five factors were F1, F9, F2, F6 and F7 ( $M=$ 4.79 to $M=4.13$ ), these five factors possessed the highest impact power on this sample's motivation; the bottom five factors were F17, F13, F8, F14, and F10 $(M=2.40$ to $M=3.45)$ these five factors possessed less or lower impact power on this sample's motivation. While the other 8 factors' mean score were from 3.46 to 4.13 means these eight factors had medium impact power on this sample's motivation.

The results of the 2 (Gender) $\mathrm{x} 4$ (Education Levels) x 4 (Belt Colors) factorial MANOVA were showed in Table 3. That were: no significant difference in the 'Education Levels' factor $(p>.05), \Lambda=.312, F=1.406$; however, significant differences were found in the 'Gender' factor $(p<.00), \Lambda=.399, F=4.442$, and in the 'Belt colors' factor $(p<.012), \Lambda=.271, F=1.619$.

According to the research design, after significant differences effects were found, an $2 \times 4$ (Gender [males, females] $\mathrm{x}$ Belt Colors [Green; Purple, Red, Black]) Multivariate Test (MANOVA) for determine where and what the students' motivations on practicing and competing taekwondo from the 'Gender' and 'Belt-colors' factors among the 18 dependents variables was further conducted; the findings were present in Table 3:

Table 3. Descriptive Statistics of Athletes/Students' Motivations on Practicing and Competing Taekwondo $(N=85$; 51 Males, 34 Females) for Different Gender and Belt-colors

\begin{tabular}{lllllll}
\hline Motivation & \multicolumn{2}{l}{ Gender - Mean (SD) } & or & \multicolumn{3}{c}{ Belt Colors- Mean $(S D)$} \\
\hline Factor & Males (51) & Females (34) & $\underline{\text { Yellow (13) }}$ & $\underline{\text { Blue }(33)}$ & $\underline{\text { Red }(25)}$ & $\underline{\text { Black }(14)}$ \\
\hline F 1 & $4.823(.433)$ & $4.735(.447)$ & $4.692(.480)$ & $4.697(.529)$ & $4.840(.374)$ & $5.000(.000)^{*}$ \\
F 2 & $4.353(.890)$ & $4.117(.728)$ & $4.076(.759)$ & $4.333(.816)^{*}$ & $4.280(.678)$ & $4.214(.949)$ \\
F 3 & $3.725(.943)$ & $3.706(.968)$ & $4.000(.816)$ & $3.575(.921)^{*}$ & $3.600(.990)$ & $4.000(.950)^{*}$ \\
F 4 & $3.862(.894)$ & $3.588(.743)^{*}$ & $3.692(.630)$ & $3.575(.791)$ & $3.680(.852)$ & $4.357(.928)^{*}$ \\
F 5 & $3.764(.924)$ & $3.735(.986)$ & $3.692(.751)$ & $3.545(.938)$ & $3.880(.953)$ & $4.071(.916)$ \\
F 6 & $4.549(.642)$ & $3.764(.741)^{*}$ & $4.000(.816)$ & $4.242(.830)^{*}$ & $3.920(.620)$ & $5.000(.000)^{*}$ \\
F 7 & $4.333(.982)$ & $4.029(.942)^{*}$ & $3.923(.987)$ & $4.242(.957)^{*}$ & $4.200(.918)$ & $4.428(.958)$ \\
F 8 & $3.745(.928)$ & $2.441(.993)^{*}$ & $3.923(.954)$ & $3.121(.987)^{*}$ & $2.800(.944)$ & $3.571(.982)^{*}$ \\
F 9 & $4.235(.885)$ & $4.323(.726)$ & $3.384(.767)$ & $4.121(.781)^{*}$ & $4.520(.653)$ & $4.071(.914)^{*}$ \\
F10 & $3.509(.958)$ & $3.352(.929)$ & $3.000(.927)$ & $3.242(.936)$ & $4.000(.727)$ & $3.357(.949)^{*}$ \\
F11 & $3.784(.971)$ & $4.117(.844)^{*}$ & $3.615(.925)$ & $4.060(.998)^{*}$ & $3.880(.971)$ & $3.928(.984)$ \\
F12 & $3.823(.865)$ & $3.411(.945)^{*}$ & $3.153(.946)$ & $3.606(.933)^{*}$ & $3.880(.725)$ & $3.857(.864)$ \\
F13 & $2.470(.936)$ & $3.705(.968)^{*}$ & $2.153(.688)$ & $3.000(.933)^{*}$ & $3.440(.949)$ & $2.785(.989)^{*}$ \\
F14 & $3.490(.982)$ & $3.294(.923)$ & $3.153(.955)$ & $3.303(.966)$ & $3.440(.979)$ & $3.857(.949)^{*}$ \\
F15 & $4.078(.988)$ & $4.205(.968)$ & $4.538(.967)$ & $4.060(.941)^{*}$ & $4.040(.968)$ & $4.071(.986)$ \\
F16 & $3.549(.947)$ & $2.441(.966)^{*}$ & $3.769(.927)$ & $3.060(.999)^{*}$ & $4.040(.449)$ & $3.642(.927)^{*}$ \\
F17 & $2.490(.988)$ & $2.264(.977)$ & $2.307(.978)$ & $2.151(.954)$ & $2.680(.936)$ & $2.571(.633)$ \\
F18 & $3.313(.787)$ & $3.676(.966)^{*}$ & $3.923(.936)$ & $3.272(.987)^{*}$ & $3.520(.918)$ & $3.357(.634)$ \\
\hline
\end{tabular}

Note. $*=$ Significant at $p<.05$ Level. There are 32 comparisons out of 54 showed significant differences at or greater than $P<.05$ Level. F1 to F18 Represent 18 different motivation factors.

As can be seenfrom Table 3 in Gender comparisons, nine out of 18 comparisons showed significant differences at or greater than .05 level; specifically males over femeles were: F4 - 'to make new friends'; F6 -'to shape the body'; F7 - 'to improve physical health'; F8 - 'to become a taekwondo professional'; F12 - 'to get the recognition from my teacher / coach'; and F16 - "to become taekwondo coach'. While specifically femeles over meles were: F11 -'to establish prestige among my classmates / friends'; F13 - 'to reduce the learning / working pressure'; and F18 - 'For self-defence'.

On the other hand, in Belt Colorscomparisons from Table 4, 22 out of 36 comparisons showed significant differences at or greater than .05 level; specifically Black over Red in F1 - 'Because taekwondo with high technical content and unique value'; Blue over Yellow in F2 - 'For the fun and get rid of boredom'; but Yellow over Blue in F3 - 'to meet friends', and Black over Red as well in F3 -'to meet friends'; Black also over Red in F4 - 'to make new friends'. Moreover, Blue over Yellow and Black over Red in F6 -'to shape the body'; Blue over Yellow in F67-'to improve physical health'; however, Yellow over Blue and Black over Red in F 8 - 'to become a taekwondo professional'; while Yellow over Blue and Red over Black in F9 - 'to foster self-esteem', Red also over Black in F10 - to improve 
my own literacy'. Furthermore, Yellow over Blue in F12 - 'to get the recognition from my teacher / coach' and F15 'to develop the skills'; and so on. No significant diffeneces among the Belt Colors as well as the Gender in F17 'In order to satisfy the will of family'. See Table 3 for details.

\section{Discussion}

The present study was designed to explore the current status of taekwondo athletes/students' participation motivations in NYC; and to examine whether differences would exist on the motivation factors or elements among the participants' gender, education levels, and Belt Colors. First, as displayed in the results section (Note. the following arrangement was in accordance with the scores to determine the order; see Table 2 for details), among the five top highest scored factors - that were F1, F9, F2, F6 and F7, these factors possessed the highest impact power on this sample's motivation. Surprisingly, these factors all belong to 'Intrinsic factors'. Second, the next 8 higher scoring factors were F16, F15, F18, F3, F4, F5, F11, and F12, wherein F16, F15, F18 belong to 'Intrinsic factors'; While F3, F4, F5, F11, and F12 belong to 'Extrinsic factors'. Third, the bottom five factors were F17, F13, F8, F14, and F10; these five factors possessed less impact power on this sample's motivation; additionally, F13 and F14 belong to 'Intrinsic factors', F17, F8 and F10 belong to 'Extrinsic factors'; therefore, in brief we can conclude that: the present study revealed the 'Intrinsic factors' possess higher impact than those of 'Extrinsic factors', and shaped these taekwondo athletes/students' motivation. The features of this sample motivation are summarized as Figure 1.

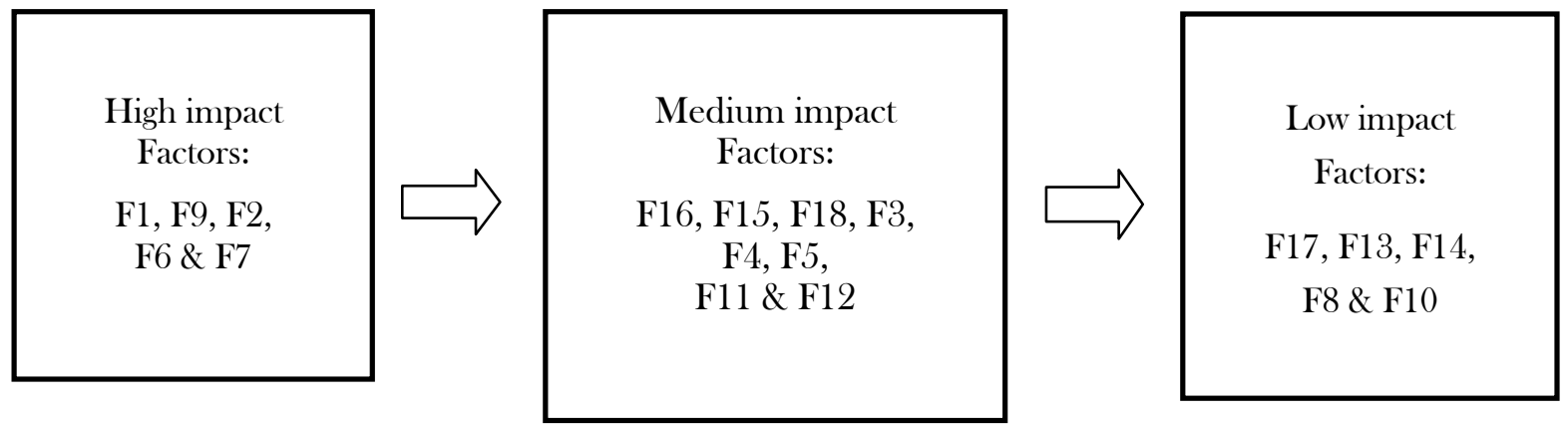

Figure 1. Three Layers of taekwondo Athletes/Students' Motivation Factors

Note. 1) Intrinsic factors $=1,2,6,7,9,13,14,15, \&$ 18. 2) Extrinsic factors $=3,4,5,8,10,11,12,16$, \& 17. And 3) These18 factors reflected the 'competence needs', the 'relatedness needs' and the 'autonomy need' in the 'self-determination theory' model interpreted by Ryan and Deci (2000).

With regard to the gender differences in the motivation factors comparisons, interesting aspects can be summarized as: First, among the total nine significant different findings, six out of nine where males scored remarkable higher than females with: F4 - 'to make new friends', F6 -'to shape the body', F7 - 'to improve physical health', F8 'become taekwondo professional athlete', 'F12 - 'to get the recognition', and F16 - "become a coach'. We believe this is a common facts that young males are keen on shaping their body, make new friends, becoming a professional athlete or coach than their female peers; therefore these MFs possessed higher impact power on males' participation motivation. Second, females scored remarkably higher than males where only three out of nine comparisons with F11 - 'establish prestige'; F13 - 'reduce the learning pressure'; and F18 - 'For self-defence'; in other words, self-defence, reduce pressures, and establish prestige are the main reasons and possess higher impact power on females' participation motivation. According to an informal after survey interview, that was true and logically made sense. Young girls or women, especially those who live in a big urban city, they are very care about their 'prestige', 'learning outcomes', and the ability of 'self-defence'. Hence, the findings on the gender differences in the participation motivation from the present study (with their living environment - a big urban city with largest population in the USA) are unique and have important referential values.

On the other hand, in regards to the motivation factors comparisons in 'Belt Colors', the interesting and unique aspects are: 1) The higher skill level the more appreciate the 'technical content and unique value' of the sport; e.g., Black belt scored higher than Red belt in F1 - 'Because taekwondo with high technical content and unique value', Black belt scored higher than Red belt in F6 -'to shape the body'. 2) The higher skill level the more value their "friendship" with classmate or teammate; e.g., Black belt scored over Red belt in F3 -'to meet friends'; and Black belt also over Red belt in F4 - 'to make new friends'. 3) Athletes possess higher skill level also have higher willing 
to become a future professional in the sport; e.g., Yellow belt scored higher than Blue belt and Black belt scored higher than Red belt in F 8 - 'to become a taekwondo professional'. However, 4) lower skill levels athletes are more carful about their self-esteem, literacy, recognition from coach, and improving their skills level; e.g., Yellow belt scored higher than Blue belt scored higher than Red belt scored higher than Black belt in F9 - 'to foster self-esteem', Red belt scored higher than Black belt in F10 -'to improve my own literacy', Yellow belt scored higher than Blue belt in F12 - 'to get the recognition from my teacher / coach' and F15 - 'to develop the skills'; and so on.

In summary, we found that there are some differences and similarities between the findings from the present study and those of the previous studies. The following are considered as some differences: a) In the research projects conducted by Ebbeck, Gibbons, and Loken-Dahle (1995), and Zaggelidis, Martinidis, and Zaggelidis (2004), their findings were: No significant differences in motivation for participation between genders and family support. b) Breese (1998) found that no significant differences among the Belt-colors rank groups in participation motivations, but different dependent upon overall time athletes involved in with those involved greater than 4 years identifying personal power and control as their primary motivation whilst those involved for less than 2 years identified fitness as their main motivation. c) In the study of Twemlow, Lerma, \& Twemlow (1996, focus on judo and karate), they found no significant differences between genders regard to participants' main participation motivations; and they summarized that 'self defense' and 'physical fitness' were two most important individual factors of motivation.

The following are considered as some similarities: a) In a study focused on collegiate taekwondo participants' motivation, Stefanek (2004) found that motivations such as fun, physical exercise, skill development, and friendship were the top four factors. Additional motives amongst the top ten identified factors were integrating and improving both mental and physical health, increasing perseverance, and reducing stress (Stefanek, 2004). b) While Zangelidis et al. (2004) found that the top three most highly rated motives by the participants were 'interest in the sports' 'health benefits' and 'character cultivation'. c) In a martial arts study, Kilpatrick, Hebert and Bartholomew (2005) concluded that reasons for people engaging in the sport relate to one's appearance and physical active lifestyle. Practicing and learning involved in taekwondo are considered beneficial to one's overall physical appearance, health and fitness. d) In other martial arts studies such as the studies of Bujak (2005) and Kusnierz (2011), they reported factors that the essential motivation for taking parting in martial arts training was to master the hand-to-hand fighting skills and self-defense rules; the martial arts training not only increased self-discipline but also improved the level of fitness and health as well; those are the especial factors that motivated the participants to keep engaging in the sports they love.

Additionally, our findings are partially consistent with the findings of Cynarski (2012) and Litwiniuk and Cynarski (2001), such as: The factors that motivate young people take part in martial arts practices are associated with the desire to improve physical fitness, acquire fighting skills, meet interesting people, and sport competition; as well as acceptance by the martial arts group (e.g., their teammates or class mates, teacher or coach).

\subsection{Limitations}

We do note several limitations of the present study. First, the size of sampling in this study was relatively small. Second, due to lack of funding support, the data collection scopes only covered two Boroughs of the choose city. Third, taekwondo instructors/coaches who might have a big impact on their athletes/students' participation motivations were not include in the objects of the study. Last, the participants in the current study were selected groups, all of them did continually participate in and did not withdraw from his/her school or club, and therefore, no withdrawal issues or practices patterns change were covered. Future study can be improved on the above limitations; by including the instructors/coaches from those participants' school or club (e.g., creating some open-ended questions for them to answer).

\subsection{Conclusion}

In summary, to conclude the key participation motivations for this sample's taekwondo athletes/students, the most important reasons or factors are the following three combination factors: a) The five intrinsic motivations that include: F1, F9, F2, F6 and F7, should be re-named as 'Value, self-esteem, enjoyment and physical needs'. b) The five extrinsic motivations that include: F15, F11, F4, F5, and F3, we re-named as 'develop the skills', 'establish prestige', 'build-up friendship', and 'contest winners'; c) Multiple intrinsic and extrinsic motivation combinations that include: F16, F8, F18, F10, F14 and F13, we re-named as 'future career', 'self-defense', and 'improve own literacy'. The two smallest influential motivational factors are: F13 'reduce working pressure' and F17 'satisfy the family's wish', as to participate in taekwondo practices and competitions, these two factors possess much less importance. 
With regard to the three specific hypotheses that guided the current study, the findings revealed that two out of the three hypotheses are not true, wherein: (1) Significant differences do exist on the motivation factors between the gender of the athlete/students, with motivation factors F4, F6, F7, F8, F12, and F16 possess significant higher impact on males than those of females; whereas motivation factors F11 - 'to establish prestige', F13 - 'to reduce pressure'; and F18 - 'for self-defense' females over those of males. (2) Significant differences do exist on the motivation factors among the Belt-colors of the athlete/students, with $2 / 3$ of the comparisons were significant differences (e.g., Black over Red in F1 - 'Because taekwondo with high technical content and unique value'; Blue over Yellow in F2 - 'For the fun and get rid of boredom'; but Yellow over Blue in F3 -'to meet friends', and Black over Red as well in F3 -'to meet friends'; Black also over Red in F4 - 'to make new friends'). (3) No significant differences, however, were found among the participants with regard to their educational levels.

\section{Acknowledgements}

The researchers of this research project wishes to express their thanks and deepest appreciation to all the participants and those instructors / coachers in the taekwondo schools / clubs. For the sharing of their motivations and the situation on took part in taekwondo practices and competitions. Without their cooperation and supporting the survey will not be possible.

The present study was supported by International Martial Arts and Combat Sports Scientific Society (IMACSSS), Poland. And it is a sub-project of Martial Arts - the social dimension of participation; the IMACSSS Project (2014 2016) under the auspices of the Division of Sociology and Anthropology and the Division of Pedagogy.

\section{References}

Breese, H. P. (1998). Participation motivation in ITFNZ Taekwon-Do: A study of the central districts region. Massey University, NZ.

Bujak, Z. (2001a). Characteristics of Taekwondo ITF. Ido - Ruch dla Kultury / Movement for Culture, 2, 33-39.

Bujak, Z. (2001b). Methods used in Taekwondo training (ITF version). Ido - Ruch dla Kultury/Movement for Culture, 2, 63-68.

Bujak, Z. (2001c). Choi Hong Hi, the contemporary legend of martial arts, Ido - Ruch dla Kultury/Movement for Culture, 2, 396-397.

Bujak, Z. (2005). Physical fitness of Taekwondo trainees and its conditioning, Ido - Ruch dla Kultury / Movement for Culture, 5, 23-29.

Capranica, L., Chiodo, S., Cortis, C., Corrado, L., Ammendolia, A., \& Tessitore, A. (2010). Scientific approaches to Olympic taekwondo: research trends. Ido - Ruch dla Kultury/Movement for Culture, 10, 73-77.

Child, D. (1990). The essentials of factor analysis, second edition. London: Cassel Educational Limited.

Choi, H. H. (1995). Taekwon-do. The Korean Art of Self Defense. International Taekwondo Federation, New Zealand.

Clough, P., Shepherd J., \& Maughan, R. (1989). Motives for participation in recreational running. Journal of Leisure Research, 21, 297-309.

Cox, R. H. (2011). Sport Psychology: Concepts and Application. Brown \& Benchmark, Dubuque.

Cynarski, W. J. (2011). Teaching of fighting arts in pedagogical and sociological perspective. Studia Sportiva, 5, 275-284.

Cynarski, W. J. (2012). Values of martial arts in the light of the anthropology of martial arts. Journal of Combat Sports and Martial Arts, 3, 1-4. http://dx.doi.org/10.5604/20815735.1047608

Cynarski, W. J. (2014a). Karate in Europe - Institutional development and changes: "karatedo wa rei ni hajimari, rei ni owaru kite wo wasuruna". Research Journal of Budo, 46, 168-183. http://dx.doi.org/10.14589/ido.14.3.2

Cynarski, W. J. (2014b). The European karate today: The opinion of experts. Ido Movement for Culture. Journal of Martial Arts Anthropology, 14, 10-21.

Cynarski, W. J., Obodynski, K., \& Zeng, Z. H. (2012). Martial arts anthropology for sport pedagogy and physical education. Romanian Journal for Multidimensional Education Lumen, 4, 133-156.

Devine, R., \& Lepisto, L. (2005). Analysis of the healthy lifestyle consumer. Journal of Consumer Marketing, 22, 
275-283. http://dx.doi.org/10.1108/07363760510611707

Ebbeck, V., Gibbons, S. L., \& Loken-Dahle, L. J. (1995). Reasons for adult participation in physical activity: an interactional approach. International Journal of Sport Psychology, 26, 262-275

Gill, D.L., Gross, J.B., \& Huddleston, S. (1983), Participation motivation in youth sports. International Journal of Sport Psychology, 14, 1-14.

Harter, S. (1981). A new self-report scale of intrinsic versus extrinsic orientation in the classroom: Motivational and informational components. Developmental $\quad$ Psychology, $\quad 17, \quad 300-312$. http://dx.doi.org/10.1037/0012-1649.17.3.300

Hartl, J., Faber, U., \& Bögle, R. (1989). Taekwondo in Westen. Interviews und Beiträge zum kulturellen Schlagabtausch. Mönchseulen-Verlag, Munich [in German].

Jones, G. W., Mackay, K. S., \& Peters, D. M. (2006). Participation motivation in martial artists in the West Midlands Region of England. Journal of Sports Science Medicine, 5, 28-34.

Kim, D., \& Back, A. (2000). The way to go: philosophy in martial arts practice. Nanam: Seoul.

Kim, S. (2010). Dr. Daeshik Kim (1934-2007), Master-teacher of martial arts from Korea. Ido - Ruch dla Kultury / Movement for Culture, 10, 163-171.

Kusnierz, C. (2011). Values associated with practicing modern karate as a form of cultivating old Japanese Bushido patterns. Ido Movement for Culture. Journal of Martial Arts Anthropology, 11, 1-5.

Lee, K. M., \& Nowicki, D. (1988). Taekwondo. Almapress, Warsaw [in Polish].

Litwiniuk, A., \& Cynarski, W.J. (2001). Motives of participation in chosen combat sports and martial arts. Ido Ruch dla Kultury / Movement for Culture, 2, 242-244.

Madis, E. (2003). The evolution of taekwondo from Japanese karate. In: T. A. Green, J. R. Svinth [eds.], Martial arts in the modern world, Praeger, Westport, 185-207.

Pieter, W., \& Bercades, L. T. (2010). Strength and power in Filipino varsity taekwondo-in. Ido - Ruch dla Kultury / Movement for Culture, 10, 67-72.

Pieter, W., \& Heijmans, J. (2000). Scientific Coaching for Olympic Taekwondo (2 ${ }^{\text {nd }}$ edn.). Meyer \& Meyer Sport, Oxford, UK.

Pintrich, P. R., \& Schunk, D. (2002). Motivation in education: Theory, research and applications $\left(2^{\text {nd }}\right.$ ed.). Upper Saddle River, NJ: Prentice-Hall.

Rosa, V. A. V. (2012). Samurais "in modern Europe: motivations and understanding of Portuguese karatekas. Ido Movement for Culture. Journal of Martial Arts Anthropology, 12, 11-19.

Ryan, R. M., \& Deci, E. L. (2000). Self-determination theory and the facilitation of intrinsic motivation, social development, and well-being. American Psychologist, $55, \quad 68-78$. http://psycnet.apa.org/doi/10.1037/0003-066X.55.1.68

Ryan, R. M., Frederick, C. M., Lepes, D., Rubio, D., \& Sheldon, K. S. (1997). Intrinsic motivation and exercise adherence. International Journal of Sports Psychology, 28, 355-354.

Rzany, R. (2002-2003). The influence of Chinese and Japanese martial arts on the origin of Korean art of taekwondo and its identity. An outline of problem. Ido-Ruch dla Kultury / Movement for Culture, 3, 56-60.

Stefanek, K.A. (2004). An exploration of participation motives among collegiate taekwondo participants. Kinesiology Publications, University of Oregon, Eugene, OR.

Taekwondo Belt System. (2015). Retrieved from http://www.taekwondo-network.com/tae-kwon-do-belt-system.html

Tedeschi, M. (2004). Taekwondo: Complete WTF forms, Weather hill Inc. Trumbull, CT.

Twemlow, S., Lerma, B., \& Twemlow, S. W. (1996). An analysis of student's reasons for studying martial arts. Perceptual and motor skills, 83, 99-103. http://dx.doi.org/10.2466/pms.1996.83.1.99

Vertonghen, J., \& Theeboom, M. (2010). The social-psychological outcomes of martial arts practice among youth: a review. Journal of Sports Science and Medicine, 9, 528-537. [PMCID: PMC3761807]

Vit, M., \& Reguli, Z. (2011). Motivation and value orientation of combative systems trainers. Ido Movement for Culture. Journal of Martial Arts Anthropology, 11, 52-59. 
Walczak, J. (2007). Original program for teaching Taekwondo in gymnasium (lower secondary school). Ido - Ruch dla Kultury / Movement for Culture, 7, 185-195.

Wasik, J. (2014). Three areas of Taekwondo identification and practice. Ido Movement for Culture. Journal of Martial Arts Anthropology, 14, 22-26.

Wikipedia. (July 25, 2015). Demographics of New York City. Retrieved from https://en.wikipedia.org/wiki/New_York_City

Witkowski, K., Cynarski, W. J., \& Blazejewski, W. (2013). Motivations and determinants underlying the practice of martial arts and combat sports. Ido Movement for Culture. Journal of Martial Arts Anthropology, 13, 17-26.

Zaggelidis, G., Martinidis, K., \& Zaggelidis, S. (2004). Comparative study of factors - motives in beginning practicing judo and karate. Physical Training: Fitness for Combative, 1, 1-8.

Zeng, Z. H., Cynarski, W. J., \& Xie, L. S. (2013). Martial Arts Anthropology, Participants' Motivation and Behaviours. Martial Arts in Chanshu: Participants' Motivation, Practice Times and Health Behaviours. Lambert Academic Publishing, Saarbrücken.

\title{
Appendix A
}

\section{ADAPTED QUESTIONNAIRE OF TAEKWONDO STUDENT’'MOTIVATION (QTKDSM)}

\author{
Adapted by Howard Z. Zeng (D.P.E.) \\ Professor of Physical Education/Sport Pedagogy \\ Brooklyn College of the City University of New York, USA
}

Direction: Dear participant! This is a survey project concerning the factors of motivation amoug various taekwondo studentsin New York City. This survey is anonymous and the information collected will be used for research purpose only. There are 28 questions in total. Read and answer every question according to your own situation and experience. It will take about 5-6 minutes to complete. Your participation will help the Taekwondo community improve their teaching/coaching and manage various competitions more effectively. Thank you!

\section{Part I. General Information}

1. What is your gender? Male __ Female

2. What is your Height (CM, please write here ___ $)$ and Weight $(\mathrm{kg}$, please write here

3. What is your age-range? (PLEAS CIRCLE ONE)
a) $\quad 10-12$
b) $\quad 13-15$
c) $\quad 16-18$
d) 19 and UP

4. How long have you officially learnt/practiced taekwondo?
a) one year
b) two years
c) three years
d) four year and more

5. What is the name of taekwondo shcool/ clubare you attending?

(Please write here: ) .

6. What school level are you currently going to?
a) Elementary school
b) Middle school
c) High school
d) Others levle of school (please specific E.g., college

7. Is your parents support you learn/practice taekwondo?
a) Very strong support
b) kind support
c) Somwhat support
d) not support

8. How many times do you practice per week?

(Please write here

9. What is your Belt Color currently? 
(Please write here

10. Where do you live during your taekwondo training/practising?
a) Dormitory
b) boarding-school
c) private house
d) with parents

\section{Part II. Motivations Questions}

$>$ What reasons or factors motive you keep practice taekwondo and take part in taekwondo competition?

Direction: There are 18 statements or factors below, answer the question by circling on each reason or factor that most fit in your situation; number 5 represents "strongly fit", number 4 represents "it fit", number 3 represents "somewhat fit", number 2 represents "little fit", and number1 represents "not fit at all".

M1. Because taekwondo have high technical content and unique value. $\quad\left(\begin{array}{lllll}5 & 4 & 3 & 2 & 1\end{array}\right)$

M2. For the fun and get rid of boredom. $\quad\left(\begin{array}{lllll}5 & 4 & 3 & 2 & 1\end{array}\right)$

M3. In order to meet friends. $\quad\left(\begin{array}{lllll}5 & 4 & 3 & 2 & 1\end{array}\right)$

M4. In order to make new friends. $\quad\left(\begin{array}{lllll}5 & 4 & 3 & 2 & 1\end{array}\right)$

M5. In order to contest winners. $\quad\left(\begin{array}{lllll}5 & 4 & 3 & 2 & 1\end{array}\right)$

M6. In order to shape the body. $\quad\left(\begin{array}{lllll}5 & 4 & 3 & 2 & 1\end{array}\right)$

M7. In order to improve physical health. $\quad\left(\begin{array}{lllll}5 & 4 & 3 & 2 & 1\end{array}\right)$

M8. For the near future may become a taekwondo professional. $\left.\quad \begin{array}{llllll}5 & 4 & 3 & 2 & 1\end{array}\right)$

M9. In order to foster self-esteem. $\quad\left(\begin{array}{lllll}5 & 4 & 3 & 2 & 1\end{array}\right)$

M10. In order to improve my own literacy. $\quad\left(\begin{array}{lllll}5 & 4 & 3 & 2 & 1\end{array}\right)$

M11. In order to establish prestige among my classmates/friends. $\left(\begin{array}{lllll}5 & 4 & 3 & 2 & 1\end{array}\right)$

M12. In order to get the recognition from my teacher/ coach. $\left.\quad \begin{array}{lllll}5 & 4 & 3 & 2 & 1\end{array}\right)$

M13. In order to reduce the learning / working pressure. $\quad\left(\begin{array}{lllll}5 & 4 & 3 & 2 & 1\end{array}\right)$

M14. In order to reduce the troubles from learning / work. $\left.\quad \begin{array}{lllll}5 & 4 & 3 & 2 & 1\end{array}\right)$

M15. In order to develop the skills. $\quad\left(\begin{array}{lllll}5 & 4 & 3 & 2 & 1\end{array}\right)$

M16. Hope to become taekwondo coach in near future. $\quad\left(\begin{array}{lllll}5 & 4 & 3 & 2 & 1\end{array}\right)$

M17. In order to satisfy the will of family. $\quad\left(\begin{array}{lllll}5 & 4 & 3 & 2 & 1\end{array}\right)$

M18. For self-defences $\quad\left(\begin{array}{lllll}5 & 4 & 3 & 2 & 1\end{array}\right)$ 


\section{Appendix B}

\section{PARTICIPANT ASSENT FORM}

\section{Title of Protocol: A SURVEYOF TAEKWONDOSTUDENT' MOTIVATION}

\section{Dear Athletes/Students,}

This is an international cooperative research project for investigating the Motivation of various taekwondo athletes/students around the world. I am an associate professor in the Department of kinesiology, Brooklyn College, CUNY. Your participation and the survey results will help the international taekwondo community improve their teaching/ coaching and manage various competitions more effectively.

You are invited to volunteer to participate in this survey by answering a questionnaire that will be administered by your instructor / coach in a classroom / gym setting; that is a paper and pencil response (take about 5-6 minutes to complete). The results of this survey will be used for writing the survey report and presentation. Your identity will remain confidential; and your responses to the questionnaire will remain anonymous. Your participation is entirely voluntary. You may say no to this invitation, and your decision will have no consequence.

If you have any questions regarding this survey, please contact Dr. Howard Z. Zeng at (718) 951-5000 ${ }^{\text {ext. } 6737 \text { at }}$ Brooklyn College.

Yes, I want to participate voluntarily in the survey. (_Yes_)

No, I will not participate in the survey. $\quad$ (No_

Date

Your participation is appreciated!

Responsible Investigator:

Dr./Prof. Howard Z. Zeng

Department of Kinesiology

Brooklyn College of the City University of New York 